\title{
Asymptotical stability of the motion of mechanical systems with partial energy dissipation
}

\author{
Volodymyr Puzyrov • Jan Awrejcewicz
}

Received: 30 May 2017 / Accepted: 9 October 2017 / Published online: 4 November 2017

(C) The Author(s) 2017. This article is an open access publication

\begin{abstract}
We consider a linear mechanical system under the action of potential, gyroscopic and dissipative (partial) forces. The classical Kelvin-Chetaev theorems are not applicable here, and another approach, which is based on Barbashin-Krasovskii theorem, is suggested. This approach is based on decomposition of the whole system and is convenient for systems of high dimension or with uncertain parameters. Some advantages of the proposed method are demonstrated by examples.
\end{abstract}

Keywords Stability - Dissipative/gyroscopic/potential forces · Kelvin-Chetaev/Barbashin-Krasovskii theorems

\section{Introduction}

The methods of the stability theory are mathematically rigorous and widely used in various applied problems.

\footnotetext{
V. Puzyrov

Department of Higher Mathematics and Methodology of Teaching Mathematics, Faculty of Mathematics and Information Technology, Vasyl Stus Donetsk National University, Vinnytsia 21021, Ukraine e-mail: doe.seldon@gmail.com

J. Awrejcewicz ( $\square)$

Department of Automation, Biomechanics and Mechatronics, Lodz University of Technology, 1/15

Stefanowski Str., Lodz 90-924, Poland

e-mail: jan.awrejcewicz@p.lodz.pl
}

The forces acting on the mechanical system often can be employed and classified according to their physical nature. The state-of-the-art classification considers potential, gyroscopic, dissipative and circulatory forces. The influence of these forces (excluding the last one) has been characterized by the following classical Kelvin-Chetaev theorems [1-3]:

Theorem 1 If the equilibrium of the mechanical system is stable under the action of potential forces only, it becomes asymptotically stable while adding dissipative forces with full dissipation.

Theorem 2 If the isolated equilibrium is unstable under the action of potential forces only, it cannot be stabilized by adding arbitrary dissipative forces with full dissipation.

Theorem 3 If the isolated equilibrium is unstable under the action of potential forces only, it remains unstable while adding arbitrary gyroscopic forces and dissipative forces with full dissipation.

Later these theorems were generalized in numerous papers, both from theoretical [4-10] and applied [11-14] points of view. Rather complete observations of references on this topic are given in [15-18]. Most of these studies considered the classical case of full energy dissipation when Rayleigh function $R$ is positive definite on all generalized velocities. However, numerous examples of mechanical systems in different areas of technology (celestial mechanics, robotics, seis- 
mology, etc.) consider the situation when dissipation is partial, i.e., function $R$ is semi-positive. As Chetaev has noted, "If the dissipation is incomplete, then the stability of equilibrium existing under certain potential forces will not be strengthened from adding such dissipative forces to the asymptotic stability" [2]. Actually, this is not quite true, because the result of the influence of such forces may vary, and whether the system becomes asymptotically stable or not strongly depends on other forces. This dependence is more complicated and less obvious than those addressed in Theorems 13. One of the first researchers, who drew attention to this, was Zajac. He noted that "In the design of attitudecontrol systems, one strives not for a positive definite damping matrix but rather for damping that affects the entire system, so that any motion induces energy dissipation" and introduced the term "pervasiveness" for this kind of damped systems [5].

Some results concerning this problem are presented in papers [19-23]. Bernstein and Bhat [24] formulated necessary and sufficient conditions for Lyapunov stability, semi-stability and asymptotic stability of matrix second-order systems with and without damping. The problem regarding stability of mechanical systems subjected to dissipative, gyroscopic, conservative/nonconservative forces has been reconsidered again by Agafonov [25]. The condition of asymptotic stability under action of the mentioned forces has been proposed as well as the estimation of the attraction domain in phase space has been formulated. The relation between stability of origin and precession systems has been derived, and examples of stabilization of the stationary motion of the balanced gimbal suspension gyro through external moments have been provided. A generalization of the Barbashin-Krasovskii theorem has been proposed by Jiang [26]. More recently, Tonkov [27] addresses the problem devoted to the BarbashinKrasovskii asymptotic stability theorem in application to control systems on smooth manifolds. Kalyakin [28] studied the autoresonance problem aimed at distinguishing solutions with unboundedly increasing amplitude. The constructed Lyapunov function allowed to investigate stability of the autoresonance with respect to perturbation of the initial data and regarding constantly acting perturbations. In this paper, we confine ourselves to autonomous linear system with potential, gyroscopic and dissipative forces. Such a system can be presented in the following form
$M \ddot{\xi}+B \dot{\xi}+K \xi=\mathbf{0}$,

$M, K, B$ are square real matrices, two first of them are symmetric and positive, $\boldsymbol{B}$ is semi-positive and always can be separated to symmetric (dissipative) and skewsymmetric (gyroscopic) components $\boldsymbol{B}=\boldsymbol{D}+\boldsymbol{G}, \boldsymbol{\xi} \in$ $\mathbb{R}^{n}$.

Our aim is to suggest the constructive approach to distinguish cases whether the stability is asymptotical with respect to all variables or not. We believe it will be effective both for systems of high dimension and systems with uncertain parameters.

\section{Preliminaries}

Moran's criterion [20] states that system (1) is asymptotically stable if and only if none of the eigenvectors $v$ of the conservative system $M G K$ lies in the null space of $\boldsymbol{D}$, that is $\boldsymbol{D} \boldsymbol{v} \neq 0$ for all eigenvectors $\boldsymbol{v}$.

Also Muller [21] has given criterion whether system (1) is pervasive from the viewpoint of the control theory

$$
\begin{gathered}
\operatorname{rank}\left[M^{-1} D,\left(M^{-1} K\right)\left(M^{-1} D\right), \ldots,\right. \\
\left.\left(M^{-1} K\right)^{n-1}\left(M^{-1} D\right)\right]=n .
\end{gathered}
$$

Both conditions are effective when matrices of (1) are numerical or a system has a few parameters. However, if the number of parameters and $n$ exceed 3-4, the verification of these conditions becomes too complicated. In this situation, some kind of decomposition may be helpful.

For our purposes, we shall use the classical Barbashin-Krasovskii theorem:

Theorem 4 [29]. Consider the ODE system

$\dot{x}=f(t, x)$,

where $\boldsymbol{f}$ is $\omega$-periodic function on $t$. Let $\boldsymbol{x}=\mathbf{0}$ be an equilibrium point for (2), and $V: \Omega \rightarrow R$ be a continuously differentiable function on a domain $\Omega \subset$ $\mathrm{R}^{n}$ containing the origin, such that

$\dot{V}(t, \boldsymbol{x}(t)) \leq 0$

in $\Omega$. Let further suppose that no other solution can stay in $S=\{\boldsymbol{x} \in \Omega: \dot{V}(\boldsymbol{x})=0\}$, other than the trivial solution $\boldsymbol{x}=\mathbf{0}$. Then, the origin is:

(A) Asymptotically stable (locally) if $V$ is positive definite;

(B) Unstable if $V$ takes negative values. 


\section{Main results}

Below we use the block notation for square matrix $\boldsymbol{P}$ of $s+l$ order in the form

$\boldsymbol{P}=\left(\begin{array}{ll}\boldsymbol{P}_{11} & \boldsymbol{P}_{12} \\ \boldsymbol{P}_{21} & \boldsymbol{P}_{22}\end{array}\right)$,

where $\boldsymbol{P}_{11}, \boldsymbol{P}_{22}-$ are square matrices of $s$ and $l$ orders, respectively, and $\boldsymbol{P}_{12}, \boldsymbol{P}_{21}-$ corresponding rectangle matrices. Also, we split the vector $\boldsymbol{\xi}$ to sub-vectors

$\boldsymbol{\xi}=\operatorname{col}(\boldsymbol{x}, \boldsymbol{y}), \quad \boldsymbol{x} \in \mathbb{R}^{s}, \quad \boldsymbol{y} \in \mathbb{R}^{l}$.

We suppose that matrix $\boldsymbol{D}=\mathbf{0}_{s} \bigoplus \operatorname{diag}\left(d_{1}\right.$, $\left.d_{2}, \ldots, d_{l}\right)$, in other words, the right lower block $\boldsymbol{D}_{22}$ is diagonalized, and three other blocks are zero matrices. Matrix $\boldsymbol{D}_{22}$ is positive. Similarly, we denote differential operators

$$
\begin{aligned}
L & =\boldsymbol{M} \frac{\mathrm{d}^{2}}{\mathrm{~d} t^{2}}+\boldsymbol{B} \frac{\mathrm{d}}{\mathrm{d} t}+\boldsymbol{K}, \\
L_{11} & =\boldsymbol{M}_{11} \frac{\mathrm{d}^{2}}{\mathrm{~d} t^{2}}+\boldsymbol{G}_{11} \frac{\mathrm{d}}{\mathrm{d} t}+\boldsymbol{K}_{11},
\end{aligned}
$$

and the corresponding lambda-matrices (matrix polynomials)

$$
\begin{aligned}
\boldsymbol{\Lambda}(\lambda) & =\boldsymbol{M} \lambda^{2}+\boldsymbol{B} \lambda+\boldsymbol{K}, \\
\boldsymbol{\Lambda}_{11}(\lambda) & =\boldsymbol{M}_{11} \lambda^{2}+\boldsymbol{G}_{11} \lambda+\boldsymbol{K}_{11} .
\end{aligned}
$$

Let $\lambda_{0}$ be some eigenvalue of $\boldsymbol{L}_{11}$, and $\boldsymbol{\beta}_{10}-$ the corresponding eigenvector, i.e.,

$\boldsymbol{\Lambda}_{11}\left(\lambda_{0}\right) \boldsymbol{\beta}_{10}=\mathbf{0}_{s}$.

Here $\mathbf{0}_{s}$ means the matrix-column with $s$ zero elements. One can introduce the equality

$$
\boldsymbol{\Lambda}_{21}\left(\lambda_{0}\right) \boldsymbol{\beta}_{10}=\mathbf{0}_{l}
$$

Theorem 5 Let us consider a mechanical system, motion equations of which are described by (1) and suppose that none of the eigenvectors of operator $\boldsymbol{L}_{11}$ satisfies condition (3). Then, adding to system an arbitrary dissipative force, which provides full dissipation on $\dot{\boldsymbol{y}}$, leads to the following results:

(I) If all eigenvalues of matrix $\boldsymbol{K}$ are positive, then equilibrium of (1) becomes asymptotically stable. Stability is exponential and uniform.

(II) If matrix $\boldsymbol{K}$ has some negative eigenvalues, then equilibrium is unstable, even if it was stabilized before by gyroscopic forces. Among particular solutions of the system, at least one has negative Lyapunov characteristic number.
Proof (I) Let us take the Lyapunov function

$V(\dot{\xi}, \xi)=\dot{\xi}^{T} \boldsymbol{M} \dot{\xi}+\xi^{T} \boldsymbol{K} \xi$

According to (1) its full time derivative is equal to $-\dot{\boldsymbol{y}}^{T} \boldsymbol{D} \dot{\boldsymbol{y}}$ and thus is non-positive. The set $S$ from Theorem 4 is defined by $\dot{\boldsymbol{y}}=\mathbf{0}$, consequently $\boldsymbol{y}=\boldsymbol{y}_{0}-$ const. Along with this, the system (1) is as follows

$\boldsymbol{M}_{11} \ddot{\boldsymbol{x}}+\boldsymbol{G}_{11} \dot{\boldsymbol{x}}+\boldsymbol{K}_{11} \boldsymbol{x}+\boldsymbol{K}_{12} \boldsymbol{y}_{0}=\mathbf{0}$,

$M_{21} \ddot{x}+G_{21} \dot{x}+K_{21} x+K_{22} y_{0}=0$.

Subsystem (4) is marginally stable with purely imaginary eigenvalues $\lambda_{j}, \bar{\lambda}_{j}(j=\overline{1 ; s})$, and the whole system (4-5) will be consistent if and only if the partial non-zero solution $\boldsymbol{x}_{\star}=\boldsymbol{\beta}_{j} e^{\lambda_{j} t}$ exists and satisfies the Eq. (5), here the $\boldsymbol{\beta}_{j}$ is corresponding eigenvector. In this case, the non-zero solution

$\boldsymbol{x}=\varepsilon \boldsymbol{x}_{\star}, \quad \boldsymbol{y}=\mathbf{0}$,

of system (1) belongs to set $S$, and $\varepsilon$ is an arbitrary small constant. With this fact, the conditions of Theorem 4 are broken, and system (1) is not pervasive; for example, solution (6) does not tend to zero as $t \rightarrow \infty$. On the other hand, if none of the eigenvalues/eigenvectors of $L_{11}$ is valid for (5), then system (4-5) is inconsistent, and the set $S$ is empty. According to Theorem 4 , in this case the trivial solution $\boldsymbol{\xi}=\mathbf{0}, \dot{\xi}=\mathbf{0}$ is asymptotically stable.

(II) If we change the status of the matrix $\boldsymbol{K}$ from "all eigenvalues are positive" to "at least one of them is negative", all previous arguments concerning solutions of (4-5) remain valid. However, the Lyapunov function $V$ can take negative values now, and paragraph (B) of Theorem 4 is involved; hence, Eq. (1) is unstable.

Example 1 Now, as a simple illustration of theorem 5, consider the 2-degree-of-freedom system. Note that necessary and sufficient conditions of asymptotic stability for such case were given in a number of papers (for instance, [30-32]), but we intend to clarify the mechanical sense of the edge between pervasive and marginally stable cases. To verify the condition (3), we need the matrix $\boldsymbol{D}$ to satisfy restrictions of Theorem 5 . As it is semi-positive, we can write

$\boldsymbol{D}=d\left(\begin{array}{cc}1 & p \\ p & p^{2}\end{array}\right), d>0, \quad\left(\boldsymbol{G}=g\left(\begin{array}{cc}0 & 1 \\ -1 & 0\end{array}\right)\right)$.

The eigenvectors of $\boldsymbol{D}$ are: $\boldsymbol{\beta}_{1}=(p,-1)^{T}, \boldsymbol{\beta}_{2}=$ $(1, p)^{T}$, and with transformation $\xi=\boldsymbol{S} \eta$ 
$=\left(\boldsymbol{\beta}_{\mathbf{1}}, \boldsymbol{\beta}_{\mathbf{2}}\right) \boldsymbol{\eta}$, we rewrite the motion equations in the form which allows to verify fulfillness of (3). Namely,

$$
\boldsymbol{K}=\left(\begin{array}{ccc}
2 & p & -1 \\
p & 2 & -1 \\
-1 & -1 & 1
\end{array}\right)
$$

$$
\begin{aligned}
\tilde{\boldsymbol{M}} & =\boldsymbol{S}^{-1} \boldsymbol{M} \boldsymbol{S} \\
& =\frac{1}{1+p^{2}}\left(\begin{array}{cc}
p^{2} m_{11}-2 p m_{12}+m_{22} & p\left(m_{11}-m_{22}\right)+m_{12}\left(p^{2}-1\right) \\
p\left(m_{11}-m_{22}\right)+m_{12}\left(p^{2}-1\right) & m_{11}+2 p m_{12}+p^{2} m_{22}
\end{array}\right),
\end{aligned}
$$

and the same representation holds for matrix $\widetilde{\boldsymbol{K}}$ (where each $m$ is replaced with $k$ ). The eigenvalues of $L_{11}$ are

$$
\pm i \sqrt{\frac{p^{2} k_{11}-2 p k_{12}+k_{22}}{p^{2} m_{11}-2 p m_{12}+m_{22}}}
$$

and to satisfy (3), we use two conditions

$\widetilde{b}_{21}=g=0$,

and $\widetilde{m}_{21} \lambda^{2}+\widetilde{k}_{21}=0$, which leads to

$\tilde{k}_{11} \tilde{m}_{12}-\tilde{m}_{11} \tilde{k}_{12}=0$.

Equality (7) means that any gyroscopic force makes the system pervasive [and is (8) fulfilled or not does not change this fact]. Along with this, if $\boldsymbol{G}=\mathbf{0}$ and coefficients of matrices $\boldsymbol{M}, \boldsymbol{B}, \boldsymbol{K}$ satisfy (8), the system is not pervasive. ${ }^{1}$ On the contrary, if $\boldsymbol{B}$ is symmetric, then equality (8) may be rewritten in a more descriptive form

$$
\left|\begin{array}{lll}
m_{11} & m_{12} & m_{22} \\
b_{11} & b_{12} & b_{22} \\
k_{11} & k_{12} & k_{22}
\end{array}\right|=0 .
$$

So, for system (1) ( $n=2)$ the presence of dissipative (partial) force leads to asymptotic stability with the exception of the case when gyroscopic force is absent and matrices $\boldsymbol{M}, \boldsymbol{D}, \boldsymbol{K}$ are linearly dependent.

Also the following circumstance connected with the role of gyroscopic forces for partial dissipative system should be emphasized. Taking into account the latter issue, we consider the following example.

Example 2 Let matrices of (1) be:

$\boldsymbol{B}=\boldsymbol{D}+\boldsymbol{G}=\left(\begin{array}{ccc}1 & -1 & 0 \\ -1 & 1 & 0 \\ 0 & 0 & 0\end{array}\right)+\left(\begin{array}{ccc}0 & g_{1} & g_{2} \\ -g_{1} & 0 & 1 \\ -g_{2} & -1 & 0\end{array}\right)$,

1 There is partial asymptotic stability with respect to $\eta_{2}$, i.e., $b_{12} \xi_{1}+b_{22} \xi_{2} \rightarrow 0$ as $t \rightarrow \infty$. and $\boldsymbol{M}=\boldsymbol{I}$, the identity matrix. Firstly, let us try the common approach. With the characteristic polynomial

$$
\begin{aligned}
\sum_{j=0}^{6} a_{j} \lambda^{j}= & \lambda^{6}+2 \lambda^{5}+\left(g_{1}^{2}+g_{2}^{2}+6\right) \lambda^{4} \\
& +\left(2 p+g_{2}^{2}+2 g_{2}+7\right) \lambda^{3} \\
& +\left(g_{1}^{2}+2 g_{1} g_{2}+2 g_{2}^{2}\right. \\
& \left.-2 p g_{2}-2 g_{1}-p^{2}+8\right) \lambda^{2} \\
& +2 p \lambda-p^{2}+2 p=0,
\end{aligned}
$$

applying the Lienard-Chipart criterion [33] one gets: Eqs. (1), (10) is asymptotically stable (while $0<p<$ $2)^{2}$ if and only if the determinant

$$
\begin{aligned}
\Delta_{5}= & 4 p^{3} g_{1}^{2}\left(g_{2}-1\right)^{2}+p^{2}\left(g_{2}-1\right)\left[16 g_{1}^{3}\right. \\
& +2 g_{1}^{2}\left(g_{2}-1\right)\left(g_{2}^{2}+2 g_{2}+5\right) \\
& \left.-8 g_{1}\left(g_{2}-1\right)^{2}+\left(g_{2}+1\right)^{2}\left(g_{2}-1\right)^{3}\right] \\
& +p\left(g_{1}+g_{2}-1\right)^{2}\left[4 g_{1}^{2}\left(g_{2}^{2}+2 g_{2}+5\right)\right. \\
& \left.-16 g_{1}\left(g_{2}-1\right)+2\left(g_{2}^{4}-4 g_{2}+3\right)\right]
\end{aligned}
$$

is positive. This expression is not very large; however, the direct analysis is not obvious. With the transformation matrix

$\widetilde{\boldsymbol{S}}=\left(\begin{array}{ccc}1 & 1 & 0 \\ -1 & 1 & 0 \\ 0 & 0 & \sqrt{2}\end{array}\right)$,

one has

$\widetilde{\boldsymbol{B}}=\left(\begin{array}{ccc}2 & g_{1} & \frac{\sqrt{2}}{2}\left(g_{2}-1\right) \\ -g_{1} & 0 & \frac{\sqrt{2}}{2}\left(g_{2}+1\right) \\ -\frac{\sqrt{2}}{2}\left(g_{2}-1\right)-\frac{\sqrt{2}}{2}\left(g_{2}+1\right) & 0\end{array}\right)$,

$\widetilde{\boldsymbol{K}}=\left(\begin{array}{ccc}2-p & 0 & 0 \\ 0 & 2+p & -\sqrt{2} \\ 0 & -\sqrt{2} & 1\end{array}\right)$

$\overline{2 \text { With } p<0}$, or $p>2$ it has at least one eigenvalue with positive real part. 
Let $\lambda_{0}$ be an eigenvalue of $L_{11}$ satisfying the equation

$\lambda^{4}+\left(\frac{1}{2} g_{2}^{2}+g_{2}+p+\frac{7}{2}\right) \lambda^{2}+p=0$

(it does not depend on $g_{1}$ ). The corresponding eigenvector $\boldsymbol{\beta}=\operatorname{col}\left(\beta_{1}, \beta_{2}\right)$, and what is more

$\beta_{2}=\frac{\sqrt{2}}{2} \beta_{1} \frac{\left(1+g_{2}\right) \lambda_{0}+2}{\lambda_{0}^{2}+1}$.

Obviously, the denominator is not equal to zero, because, according to (12), it would lead to

$\frac{1}{2} g_{2}^{2}+g_{2}+\frac{5}{2}=0$,

which is not possible for any real value of $g_{2}$. Therefore, equality (3) brings the following condition

$\beta_{1}\left[g_{1}+\frac{1}{2}\left(g_{2}-1\right) \frac{\left(1+g_{2}\right) \lambda_{0}+2}{\lambda_{0}^{2}+1}\right]=0$.

As $\lambda_{0}$ is a purely imaginary number, it leads to

$g_{2}^{2}-1=0, \quad g_{1}\left(\lambda_{0}^{2}+1\right)+g_{2}-1=0$.

So, if both equalities (13) are fulfilled, there is no complete asymptotical stability (probably, partial) $-L$ has purely imaginary eigenvalues. If at least one of these equalities is broken, then $L$ is asymptotically stable. In contradiction to (11), equalities (13) can be easily analyzed: (a) the first case is $g_{2}=1$, and then $g_{1}=0$;

(b) the second case is $g_{2}=-1$, and then $g_{1}=$ $2 /\left(\lambda_{0}^{2}+1\right)$, or $\lambda_{0}^{2}=2 / g_{1}-1$ and with (12):

$p=\frac{\lambda_{0}^{2}\left(\lambda_{0}^{2}+3\right)}{\lambda_{0}^{2}+1}$

we have the binding between $p$ and $g_{1}$. Finally, this case yields

$g_{2}=-1, p=\frac{\left(g_{1}-2\right)\left(g_{1}+1\right)}{g_{1}}(0<p<2)$.

Recall that in the case when $\boldsymbol{D}$ is positive, the supplementation of the MDK-system with any matrix $\boldsymbol{G}$ stores asymptotic stability property. When dissipation is incomplete, this property remains valid only for $\boldsymbol{y}$ coordinates. The presence of $\boldsymbol{G}(\boldsymbol{x})$ force may bring the residual motion.
Remark 1 Not only the rigorous analysis of (11) is difficult, but even the geometrical (numerical) way is not reliable. We have tried to plot the set $\Delta_{5}\left(g_{1}, g_{2}, p\right)=$ 0 with Maple/Mathematica, and the results were deteriorated-although the line (a) was caught, but illegible surfaces were plotted at the same time some. As it follows from (14), the required set is a part of a curve-not surface. Conditions (14) can be easily verified numerically, for example, with

$g_{2}=-1, g_{1}=\frac{7}{3}, p=\frac{10}{21}$,

the characteristic polynomial has roots $\pm l \sqrt{7} / 7$ and with $g_{2}=-1, g_{1}=2.333, p=0.476$ all real parts are negative.

\section{Another method of verification of condition (3)}

For some reasons, the methods based on the use of eigenvectors technique as well as diagonalization of the matrix $\boldsymbol{D}$ seem to be inconvenient. There is no problem to suggest another way for verification of equality (3). Actually, let matrix $\boldsymbol{D}$ of order $j \leq n$ has rank $l<$ $j$, and $\xi_{s_{1}}, \ldots, \xi_{s_{j-l}}\left(1 \leq s_{1}<\cdots<s_{j-l} \leq n\right)$ is some fundamental ${ }^{3}$ system of solutions for linear homogeneous system

$\boldsymbol{D} \xi=\mathbf{0} \quad\left(\xi \in \mathbb{R}^{j}\right)$.

Then, any solution of (15) can be given in the following form

$\xi_{s_{q}}=\alpha_{1 q} \xi_{s_{1}}+\cdots+\alpha_{l q} \xi_{s_{j-l}}(q=\overline{1 ; j})$,

where $\alpha_{1 q}, \ldots, \alpha_{l q}$ are some real constants. As it follows from the proof of Theorem 5, the emptiness of the set $S$ depends on whether some solutions (15) satisfy the system (7)-(8) or not. Eliminating $j$ variables from (1) with (16) and denoting renewed matrices as $\boldsymbol{M}^{\star}, \boldsymbol{G}^{\star}, \boldsymbol{K}^{\star}$ (these matrices are rectangular now), we get necessary and sufficient conditions of consistency the algebraic system

$\left(\boldsymbol{M}^{\star} \lambda_{0}^{2}+\boldsymbol{G}^{\star} \lambda_{0}+\boldsymbol{K}^{\star}\right) \xi^{\star}=\mathbf{0}\left(\xi^{\star} \in \mathbb{R}^{n-l}\right)$

which is tantamount to (3), and here $\lambda_{0}$ stands for some purely imaginary number. The last system is the system

3 Maximal linearly independent system of solutions. 
of $n$ homogeneous linear equations with $n-l$ variables, and the matrix $\boldsymbol{\Lambda}^{\star}\left(\lambda_{0}\right)$. This system is consistent if and only if

$\operatorname{rank} \boldsymbol{\Lambda}^{\star}\left(\lambda_{0}\right)<n-l$.

To illustrate this approach, let us study back the Example 1. Here $j=n=2, l=1$. With $\xi_{1}=-p \xi_{2}$ we get the matrix $\boldsymbol{\Lambda}^{\star}$ in the following form $\left(\begin{array}{c}\left(-p m_{11}+m_{12}\right) \lambda_{0}^{2}+g \lambda_{0}-p k_{11}+k_{12} \\ \left(-p m_{12}+m_{22}\right) \lambda_{0}^{2}+p g \lambda_{0}-p k_{12}+k_{22}\end{array}\right)$.

To satisfy (18), the rank of the matrix must be less then 1. In other words, both elements of matrix are equal to zero. As $\lambda_{0}$ is purely imaginary, we immediately conclude that $g=0$. From linear dependence of the elements of matrix with respect to $\lambda_{0}^{2}$, we get

$$
\begin{aligned}
& \left(-p m_{11}+m_{12}\right)\left(-p k_{12}+k_{22}\right)=\left(-p m_{12}\right. \\
& \left.\quad+m_{22}\right)\left(-p k_{11}+k_{12}\right) .
\end{aligned}
$$

The last can be easily rewritten in the form (9), as $b_{12}=p b_{11}, b_{22}=p^{2} b_{11}$.

The same procedure applied to example $2(n=$ $3, j=2, l=1)$ leads to :

$$
\xi_{1}=\xi_{2}, \quad \Lambda^{\star}\left(\lambda_{0}\right)
$$

$$
\begin{aligned}
= & \left(\begin{array}{cc}
\lambda_{0}^{2}+g_{1} \lambda_{0}+2+p & g_{2} \lambda_{0}-1 \\
\lambda_{0}^{2}-g_{1} \lambda_{0}+2+p & \lambda_{0}-1 \\
-\left(g_{2}+1\right) \lambda_{0}-2 & \lambda_{0}^{2}+1
\end{array}\right) \\
& \sim\left(\begin{array}{cc}
\lambda_{0}^{2}+g_{1} \lambda_{0}+2+p & g_{2} \lambda_{0}-1 \\
-2 g_{1} \lambda_{0} & \lambda_{0}\left(1-g_{2}\right) \\
-\left(g_{2}+1\right) \lambda_{0}-2 & \lambda_{0}^{2}+1
\end{array}\right) .
\end{aligned}
$$

Its rank must be less then 2 , so all minors of the second order are equal to zero. It follows that

$$
\begin{array}{r}
\left(1-g_{2}\right)\left(\lambda_{0}^{2}+2+p\right)+g_{1}\left[\left(1+g_{2}\right) \lambda_{0}-2\right]=0, \\
2 g_{1}\left(\lambda_{0}^{2}+1\right)+\left(g_{2}^{2}-1\right) \lambda_{0}+2\left(g_{2}-1\right)=0 .
\end{array}
$$

From the second equality, we have $g_{2}= \pm 1$. If $g_{2}=1$, then from the first equality (19) one gets $g_{1}=$ 0 . If $g_{2}=-1$, we come to condition (14).

This technique seems simpler than the one proposed in Sect. 3; however, it strongly depends on the specific circumstances of the investigated system.

\section{Nonlinear systems}

We consider the motion of a holonomic mechanical system subject to stationary, ideal constraints. The position of this system is specified by $m+k$ generalized coordinates with the first $m$ of them $q_{1}, \ldots, q_{m}$ being positional and $r_{1}, \ldots, r_{k}$ being cyclic. The equations of motion of the system are written in the Lagrange form

$\frac{d}{d t} \frac{\partial \mathcal{K}}{\partial \dot{q}_{j}}-\frac{\partial \mathcal{K}}{\partial q_{j}}=\frac{\partial \Pi}{\partial q_{j}}+Q_{j}(j=1, \ldots, m+k)$,

where $\mathcal{K}, \Pi$ are the kinetic and potential energies of the system, $Q_{j}$ represent the generalized non-potential forces. Expression for kinetic energy can be written as

$$
\begin{aligned}
\mathcal{K}(\boldsymbol{q}, \dot{\boldsymbol{q}}, \dot{\boldsymbol{r}})= & \frac{1}{2} \dot{\boldsymbol{q}}^{T} \widetilde{\boldsymbol{A}}(\boldsymbol{q}) \dot{\boldsymbol{q}}+\dot{\boldsymbol{r}}^{T} \widetilde{\boldsymbol{B}}(\boldsymbol{q}) \dot{\boldsymbol{q}}+\frac{1}{2} \dot{\boldsymbol{r}}^{T} \widetilde{\boldsymbol{C}}(\boldsymbol{r}) \dot{\boldsymbol{r}} \\
= & \frac{1}{2}\langle\widetilde{\boldsymbol{A}}(\boldsymbol{q}) \dot{\boldsymbol{q}}+\widetilde{\boldsymbol{B}}(\boldsymbol{q}) \dot{\boldsymbol{r}}, \dot{\boldsymbol{q}}\rangle \\
& +\frac{1}{2}\langle\widetilde{\boldsymbol{B}}(\boldsymbol{q}) \dot{\boldsymbol{q}}+\widetilde{\boldsymbol{C}}(\boldsymbol{q}) \dot{\boldsymbol{r}}, \dot{\boldsymbol{r}}\rangle
\end{aligned}
$$

Here $\widetilde{\boldsymbol{A}}, \widetilde{\boldsymbol{C}}$ are square, symmetric, positive matrices of orders $m$ and $l$, respectively; $\widetilde{\boldsymbol{B}}$ stands for the rectangular matrix of order $k \times m$. It is supposed that all three matrices have continuous partial derivatives of the second order, the upper index $T$ denotes the transpose, and oblique brackets denote the scalar product.

According to the definition of the cyclic coordinates, we have $\widetilde{\boldsymbol{B}} \dot{\boldsymbol{q}}+\widetilde{\boldsymbol{C}} \dot{\boldsymbol{r}}=\boldsymbol{\gamma}$, where $\boldsymbol{\gamma}$ is a matrix-column of cyclic constants. Expressing $\dot{\boldsymbol{r}}$ and conveying it to (21), we write down the Routh function

$$
\begin{aligned}
\mathcal{R}= & K-\dot{\boldsymbol{r}}^{T} \boldsymbol{\gamma}=\frac{1}{2} \dot{\boldsymbol{q}}^{T}\left(\widetilde{\boldsymbol{A}}-\widetilde{\boldsymbol{B}}^{T} \widetilde{\boldsymbol{C}}^{-1} \widetilde{\boldsymbol{B}}\right) \dot{\boldsymbol{q}} \\
& +\boldsymbol{\gamma}^{T} \widetilde{\boldsymbol{C}}^{-1} \widetilde{\boldsymbol{B}} \dot{\boldsymbol{q}}-\frac{1}{2} \boldsymbol{\gamma}^{T} \widetilde{\boldsymbol{C}}^{-1} \boldsymbol{\gamma},
\end{aligned}
$$

which leads to the Routh kinetic potential

$$
\begin{aligned}
L_{\mathcal{R}} & =\mathcal{R}-\Pi=\frac{1}{2} \dot{\boldsymbol{q}}^{T} \boldsymbol{A}(\boldsymbol{q}) \dot{\boldsymbol{q}}+\boldsymbol{B}(\boldsymbol{q}, \boldsymbol{\gamma}) \dot{\boldsymbol{q}}+W(\boldsymbol{q}, \boldsymbol{\gamma}) \\
\boldsymbol{A} & =\widetilde{\boldsymbol{A}}-\widetilde{\boldsymbol{B}}^{T} \widetilde{\boldsymbol{C}}^{-1} \widetilde{\boldsymbol{B}} \\
\boldsymbol{B} & =\boldsymbol{\beta}^{T} \widetilde{\boldsymbol{C}}^{-1} \widetilde{\boldsymbol{B}}, \quad W=\Pi+\frac{1}{2} \boldsymbol{\gamma}^{T} \widetilde{\boldsymbol{C}}^{-1} \boldsymbol{\gamma}
\end{aligned}
$$

Thus, the motion equations can be written as Routh equations

$\frac{d}{d t} \frac{\partial L_{R}}{\partial \dot{q}_{j}}-\frac{\partial L_{R}}{\partial q_{j}}=Q_{j}(j=\overline{1, m})$,

or in the counter part explicit form 
$\boldsymbol{A} \ddot{\boldsymbol{q}}+\boldsymbol{B}^{\star} \dot{\boldsymbol{q}}+\left(\dot{\boldsymbol{q}}^{T} \boldsymbol{D}^{1}(\boldsymbol{q}) \dot{\boldsymbol{q}}, \ldots, \dot{\boldsymbol{q}}^{T} \boldsymbol{D}^{n}(\boldsymbol{q}) \dot{\boldsymbol{q}}\right)+\frac{\partial W}{\partial \boldsymbol{q}}=\boldsymbol{Q}$.

Here $\boldsymbol{B}^{\star}$ is a skew-symmetric matrix, and

$$
\begin{aligned}
b_{k j}^{\star} & =\frac{\partial b_{k}}{\partial q_{j}}-\frac{\partial b_{j}}{\partial q_{k}}, \\
d_{j k}^{s} & =a_{s k}^{j}+a_{s j}^{k}-a_{j k}^{s}(j \neq k), \\
d_{j j}^{s} & =a_{s j}^{j}-\frac{1}{2} a_{j j}^{s} .
\end{aligned}
$$

Stationary motions of (20) are governed by equilibrium positions of (24). The last one can be determined from the following condition

$$
\frac{\partial W}{\partial \boldsymbol{q}}=\mathbf{0},
$$

Suppose that (26) determines the equilibrium state

$\boldsymbol{q}=\boldsymbol{q}_{0}, \dot{\boldsymbol{q}}=\mathbf{0}$,

the matrix $Q$ represents dissipative forces and is semipositive in the neighborhood of (27). Then equations of perturbed motion $\boldsymbol{q}=\boldsymbol{q}_{0}+\boldsymbol{\xi}, \dot{\boldsymbol{q}}=\dot{\boldsymbol{\xi}}$ are

$$
M \ddot{\xi}+(D+G) \dot{\xi}+K \xi=N(\dot{\xi}, \xi),
$$

where

$$
\boldsymbol{M}=\boldsymbol{A}\left(\boldsymbol{q}_{0}\right), \quad \boldsymbol{G}=\boldsymbol{B}^{\star}\left(\boldsymbol{q}_{0}\right), \quad \boldsymbol{K}=\left.\frac{\partial^{2} W}{\partial \boldsymbol{q}^{2}}\right|_{\boldsymbol{q}=\boldsymbol{q}_{0}},
$$

and $N(\dot{\xi}, \xi)$ comprises all nonlinear terms of the system.

Let the linear part of (28) satisfies assumptions of Theorem 5. If the zero solution is asymptotically stable, then all Lyapunov characteristic exponents are negative, and according to his stability theorem on the first approximation [34], this entails the asymptotical stability for nonlinear system. In the same way, if the linear system is unstable, then at least one characteristic exponent is positive, and this leads to instability of zero solution of the system (28). Thus, involving the denotations of Sect. 3, and above-mentioned arguments, we can formulate
Theorem 6 Assume that the linear part of equations (28) satisfies the preconditions of Theorem 5 and none of the eigenvectors of linear operator $\boldsymbol{L}_{11}$ satisfies the condition

$\boldsymbol{\Lambda}_{21}\left(\lambda_{0}\right) \boldsymbol{\beta}_{10}=\mathbf{0}_{l}$.

Then:

(1) If all eigenvalues of $\boldsymbol{K}$ are positive, the stationary motion of the mechanical system corresponding to (28) is uniformly and asymptotically stable with respect to positional coordinates and their time derivatives. Motion is stable with respect to cyclic velocities and then tends to some constant values as $t \rightarrow \infty$.

(2) If matrix $\boldsymbol{K}$ has at least one negative eigenvalue, then the motion is unstable.

Remark 2 Once again, condition (18) can be used instead of (3). It is more convenient if the matrix $\boldsymbol{D}$ is not diagonalized.

Remark 3 Function $N$ can be periodic or quasi-periodic regarding time. The statement of the theorem remains valid.

Below we illustrate the use of the theorem by taking into account two mechanical systems.

Example 3 The double pendulum with attached mass.

The double pendulum model [35] is widely used in various areas of science and technology (mechanics, physics, engineering, biomechanics, medicine, sports, etc.). One of the main reasons is the "leap" in complexity of its dynamics compared with a simple pendulum. The double pendulum behaves in a different way. If its oscillations are small, the double pendulum demonstrates the phenomenon of beats. The character of oscillations of the pendulums changes radically with increasing energy, i.e., the oscillations become chaotic $[36,37]$. This means stabilization of oscillations of the pendulum is quite a challenging problem.

Consider the double pendulum with distributed mass [38] in a gravitational field (Fig. 1). It has a fixed point $O$, and $C_{1}, C_{2}$ are the mass centers of the first and second limbs, respectively. A dynamic absorber with stiffness $k$ and damping coefficient $h$ is attached to the second limb. The absorber oscillates along the axis $\mathrm{O}_{2} x^{\prime}$, which is orthogonal to the line $O_{1} C_{2}$ and intersects it in the point $\mathrm{O}_{2}$. Pivots in the points $O, O_{1}$ are assumed to be frictionless. 


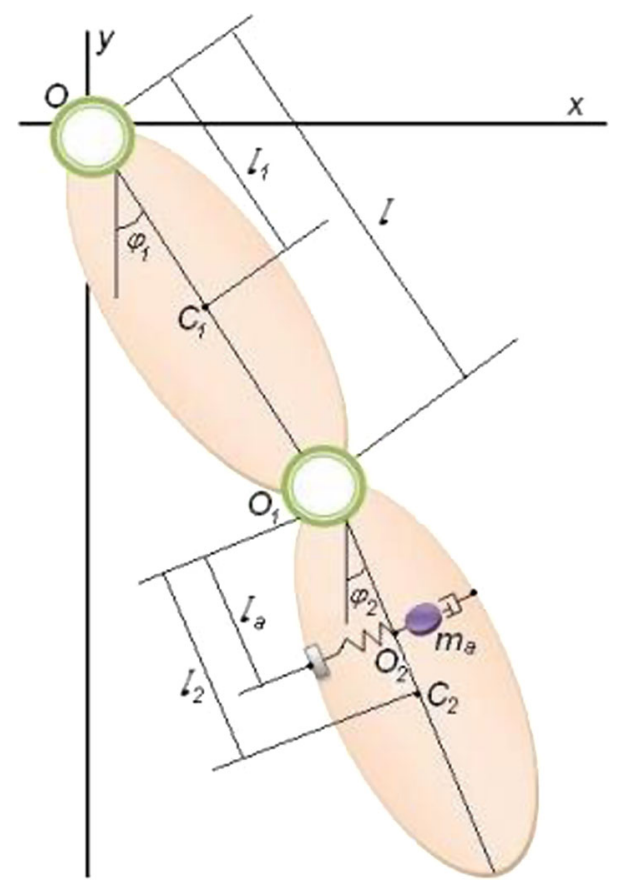

Fig. 1 Double pendulum with absorber attached

Verification of the validity of Theorem's 6 conditions by (18) yields that the rank of matrix
(For reasons of convenience, the symbol " " is neglected in elements of $\boldsymbol{\Lambda}^{\star}$, and the matrices $\boldsymbol{M}, \boldsymbol{K}$ are given in "Appendix").

Therefore, all minors of the second order have to be zeros. From the second and the third strings of the matrix we get $\lambda^{4}\left(\widetilde{I}_{2}-\widetilde{l}_{a}\right)=0$. Since $\lambda \neq 0$ (matrix $\boldsymbol{K}$ is positive), the equilibrium is asymptotically stable if $\widetilde{I}_{2} \neq \widetilde{l}_{a}$. With formulas (31) this condition reads $I_{2} \neq m_{2} l_{2} l_{a}$ or

$l_{a} \neq \frac{I_{2}}{m_{2} l_{2}}$,

and it establishes the restriction for placement of a device inside the limb of pendulum. It is remarkable that (32) does not depend on parameters of absorber $m_{a}, k, h$, but only on $l_{a}$. In other words, it reveals the "dead point" on the axis $O_{1} C_{2}$, i.e., the position for $O_{2}$ which does not result with stabilization of pendulum oscillations. This fact can be easily justified by numerical calculus: for example, considering the following parameters

$$
\begin{aligned}
& \widetilde{I}_{1}=3, \widetilde{I}_{2}=2, \widetilde{m}_{1}=\frac{2}{3}, \widetilde{l}_{1}=1, \widetilde{l}_{a}=\widetilde{I}_{2}=2, \\
& \tilde{m}_{a}=\frac{1}{3}, \widetilde{h}=\frac{1}{2}, \widetilde{k}=2, \widetilde{l}=2
\end{aligned}
$$

$\boldsymbol{\Lambda}^{\star}=\left(\begin{array}{cc}{\left[I_{1}+l^{2}\left(1+m_{a}\right)\right] \lambda^{2}+l\left(1+m_{a}\right)+m_{1} l_{1}} & l\left(1+m_{a} l_{a}\right) \lambda^{2} \\ l\left(1+m_{a} l_{a}\right) \lambda^{2} & {\left[I_{2}+m_{a} l_{a}^{2}\right] \lambda^{2}+1+m_{a} l_{a}} \\ m_{a} l \lambda^{2} & m_{a} l_{a} \lambda^{2}+m_{a}\end{array}\right)$

is equal to 1 . The following notation has been employed

$\widetilde{I}_{s}=\frac{I_{s}}{m_{2} l_{2}^{2}}(s=1,2), \tilde{m}_{1}=\frac{m_{1}}{m_{2}}, \tilde{m}_{a}=\frac{m_{a}}{m_{2}}$,

$\tilde{l}_{1}=\frac{l_{1}}{l_{2}}, \tilde{l}=\frac{l}{l_{2}}, \tilde{l}_{a}=\frac{l_{a}}{l_{2}}$,

$\tau=\sqrt{\frac{g}{l_{2}}} t, \tilde{k}=\frac{k l_{2}}{m_{2} g}, \widetilde{h}=\frac{h}{m_{2}}, \tilde{u}=\frac{u}{l_{2}}$,

where $m_{1}, m_{2}$ are the masses of the first and second limbs, respectively; $m_{a}$ is the mass of the absorber; $I_{1}, I_{2}$ are their moments of inertia with respect to poles $O, O_{1}$, respectively; $\varphi_{1}, \varphi_{2}$ are the angles of deflection of the pendulum limbs about a vertical axis; $l$ is the length of the first limb; $l_{j}(j=1,2)$ are the distances from the suspension point of the $j$-link to its mass center. the eigenvalues become:

$$
\begin{aligned}
& \pm \frac{1}{6} i \sqrt{27-3 \sqrt{33}}, \pm \frac{1}{6} i \sqrt{27+3 \sqrt{33}} \\
& -\frac{5}{12} \pm \frac{1}{12} i \sqrt{407}
\end{aligned}
$$

If the value of $\widetilde{l}_{a}$ varies a little, four pure imaginary roots acquire small negative real parts.

In Figs. 2 and 3 we show the results of the numerical simulations in form of projections of the phase trajectories on coordinate planes. Both trajectories were calculated with identical values of mechanical parameters on the time interval $\tau \in[0,150]$. The difference of trajectories character is connected with phase shift of perturbations of each limb at the beginning of the motion. In Fig. 2 this shift is small $\left(\varphi_{1}(0) \cdot \varphi_{2}(0)>0\right)$ and in Fig. 3 the shift is big, since the limbs are in antiphase at the initial time instant. By this reason, in the 

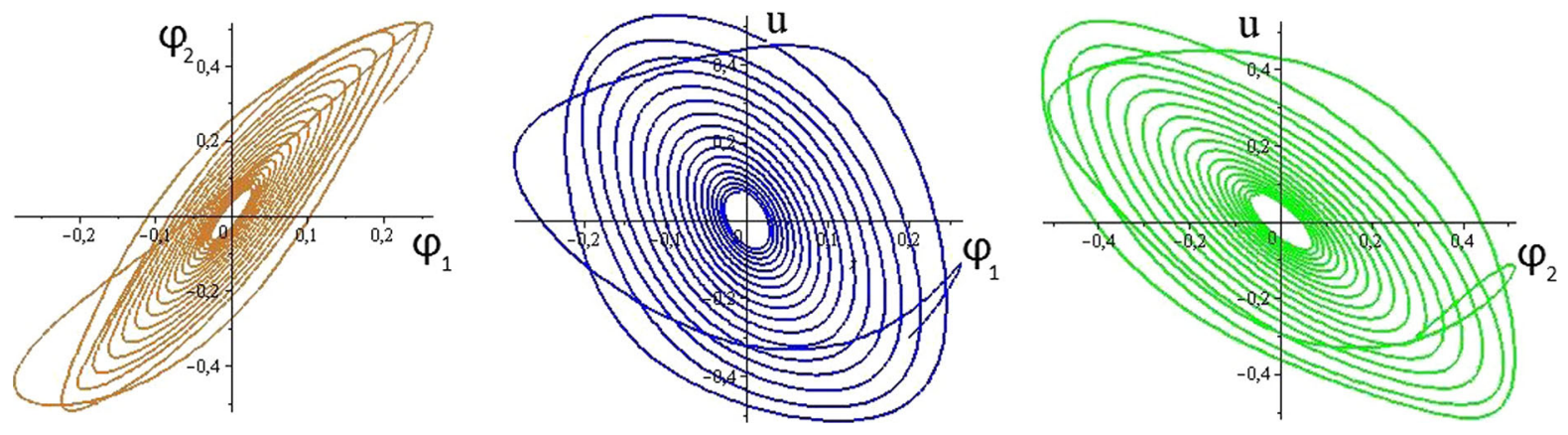

Fig. 2 Projections of the phase trajectory. $P_{0}(0.2,0.3,-0.3)$ is the initial point
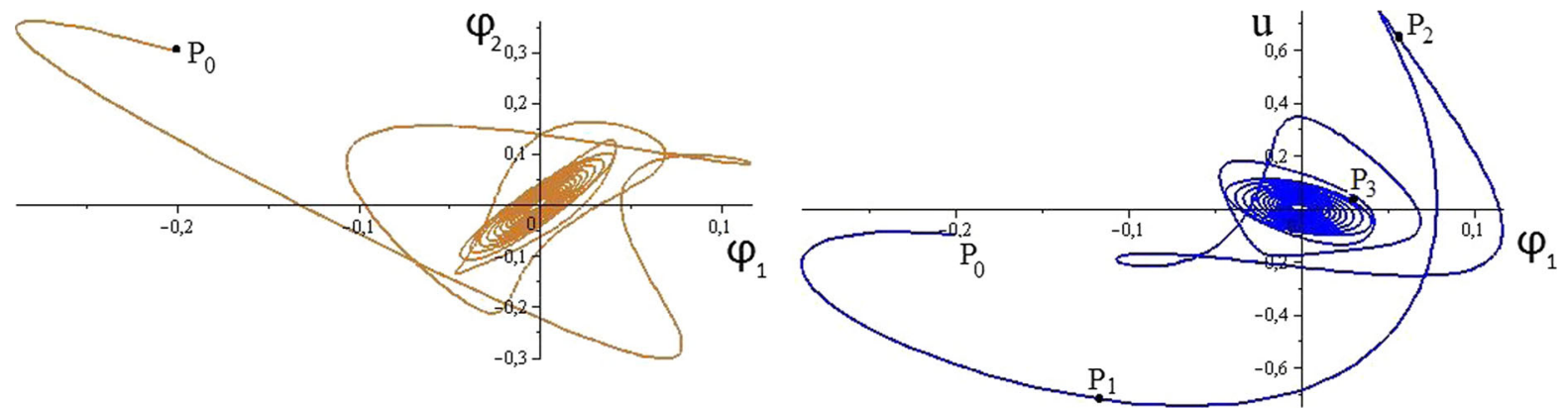

Fig. 3 Projections of the phase trajectory. $P_{1}: \tau=2 ; P_{2}: \tau=5 ; P_{3}: \tau=30$.

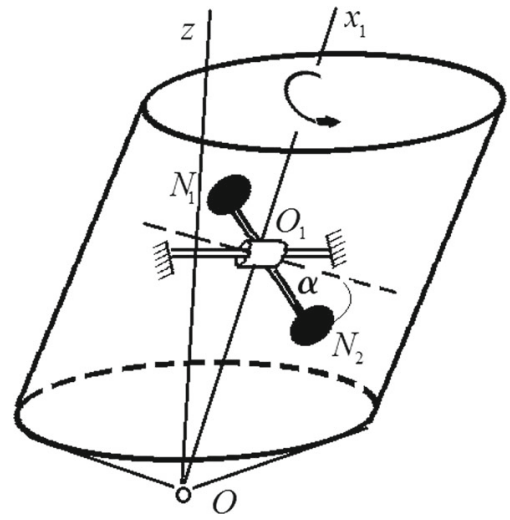

Fig. 4 Rigid body with a damper-balancer

second case the system needs more time $(\approx 30 \tau$ - seconds) to exhibit "smooth" oscillations of both limbs, and the absorber transfers more energy (amplitude of its oscillations increases several times) to recover the steady motion of the system. In both cases the oscillations of the limbs synchronize in some period of time (the spiral part of trajectory).

Example 4 Lagrange's gyroscope with damperbalancer
As a second example of the Theorem 6 application, we consider the stabilization problem for the steadystate motion (permanent rotation) of dynamically symmetric rigid body with fixed point $O$ (Fig. 4). Let $O x y z$ be an inertial coordinate system, and the system $O x_{1} y_{1} z_{1}$ be connected with the rigid body, whereas $O x_{1}$ stands for the axis of symmetry. Inside the body there is installed a balancer-a weightless rod $N_{1} N_{2}$ with two masses $m$ at the ends. This rod intersects the axis of symmetry of the body at point $O_{1}$. The balancer is mounted by viscoelastic torsion spring hinge, and $\varkappa, \hbar$ are its coefficients of stiffness and viscosity, respectively. The axis of the hinge is collinear to $O y_{1}$, so the balancer can oscillate around the point $O_{1}$ in the plane $O x_{1} z_{1}$. The length of the rod is $2 R$, and $O_{1}$ is its midpoint. As generalized coordinates we choose Euler angles $\theta, \varphi, \psi$, which describe the position of connected coordinate system with respect to inertial one, and the angle $\alpha$ between axis of the balancer and $O z_{1}$.

The motion equations and auxiliary formulas are presented in "Appendix B". The matrices of (29) have the following form 


$$
\begin{aligned}
\boldsymbol{M} & =\widetilde{\boldsymbol{A}}\left(\boldsymbol{q}_{0}\right)=\left(\begin{array}{ccc}
1 & 0 & 0 \\
0 & 1+\mu-\mu \\
0 & -\mu & \mu
\end{array}\right), \boldsymbol{D}=\operatorname{diag}(0,0, h), \\
\boldsymbol{G} & =\left(\begin{array}{ccc}
0 & 2-a & 0 \\
a-2 & 0 & 0 \\
0 & 0 & 0
\end{array}\right), \\
\boldsymbol{K} & =\left(\begin{array}{rcc}
a-b-1 & 0 & 0 \\
0 & a-b-1+\mu-\mu \\
0 & -\mu & \kappa
\end{array}\right) .
\end{aligned}
$$

Obviously, the matrix $\boldsymbol{M}$ is positive, and $\boldsymbol{K}$ is positive if and only if the inequalities

$a-b-1>0, \kappa>\frac{\mu^{2}}{a-b-1+\mu}$

hold. Then, one can easily see that

$\operatorname{rank}\left(\begin{array}{cc}\lambda^{2}+a-b-1 & (2-a) \lambda \\ (a-2) \lambda & (1+\mu) \lambda^{2}+a-b-1 \\ -\mu \lambda^{2} & -\mu\end{array}\right)>1$.

In fact, to counteract this, all minors of the second order have to be equal to zero, and this means that $\lambda$ is a purely imaginary number, as $\operatorname{det}\left(\boldsymbol{M}_{11} \lambda^{2}+\boldsymbol{G}_{11} \lambda+\right.$ $\left.\boldsymbol{K}_{11}\right)=0$. However in this case the minor

$\left(\begin{array}{cc}\lambda^{2}+a-b-1 & (2-a) \lambda \\ -\mu \lambda^{2} & -\mu\end{array}\right)$

may be equal to zero only if $a=2$, which cannot be realized due to condition $I_{1}<2 I_{2}$ (restrictions of triangle on moments of inertia). Consequently, the permanent rotations of the gyro are asymptotically stable if inequalities (33) are fulfilled. The first of those conditions reads $I_{1}+2 m R^{2}>I_{2}+2 m l_{1}^{2}$ and means that a body with a "frozen" balancer has oblate inertia ellipsoid. The second inequality sets a limit from below on the stiffness of the hinge.

\section{Discussion and concluding remarks}

We have studied the stability problem for linear mechanical system subjected to the influence of potential, gyroscopic and dissipative (partial) forces. Such a situation is more confusing than the case of complete dissipation. The latter allows one to solve the problem in a qualitative manner, i.e., by analysis of potential (or potential and gyroscopic) forces only, and conclusion does not depend on the quantitative nature of forces. In other words, only signs of matrix eigenvalues are important, not their exact values or connections between them.

While dissipation is incomplete, the results of Kelvin-Chetaev theorems mostly persist, excluding some special relations between quantitative values that characterize the forces (some surfaces in space of mechanical parameters). A way of finding these relations is proposed by formulas (3) and (18).

We note that the proposed approach is of a qualitative nature and does not allow to directly assess the quantitative characteristics of the system, i.e., its frequency, amplitude, decay rate. This is a certain drawback; however, the procedure for verifying the feasibility of these conditions is fairly simple. This procedure can be adapted to estimate the rate of damping of perturbed solutions, although it becomes much more cumbersome. For this reason, these issues are not discussed in the present paper.

As can be seen from the statements of Theorems 5 and 6 , the magnitude of the damping force does not affect the fact of stabilization of the motion of the system. In fact, this indicator is important for applications, since too little dissipative force does not allow to ensure a sufficient rate of attenuation of oscillations in the dynamical system. For instance, Fig. 5 shows the dependences for $\varphi_{2}(\tau)$ from Sect. 2 (double pendulum) for the following values of the parameters:

$\widetilde{I}_{1}=3, \widetilde{I}_{2}=2, \tilde{m}_{1}=1.3, \tilde{m}_{a}=0.3, \tilde{l}=2, \tilde{l}_{1}=1$, $\widetilde{l}_{a}=0.3, \widetilde{h}=(0.1 ; 0.6 ; 1.6), \widetilde{k}=0.8$.

It is not difficult to see that the damping is practically absent in Fig. 5a and c.

Another remarkable fact is that in the case when condition (3) or (18) holds, this does not prevent the asymptotic stability of equilibrium for a nonlinear system. The linear approximation has purely imaginary roots, and we get the critical case in Lyapunov sense $[39,40]$. For example, such a situation takes place for the system shown in Fig. 6. To prove the asymptotic stability, the Lyapunov function $V$ can be constructed. This function is the sum of positively defined quadratic form and form of the fourth order and has a negative derivative with respect to time. Basically, this procedure is not difficult, but it leads to extremely huge analytical expressions for the coefficients of the function $V$ (and its derivative), and is not discussed here. 


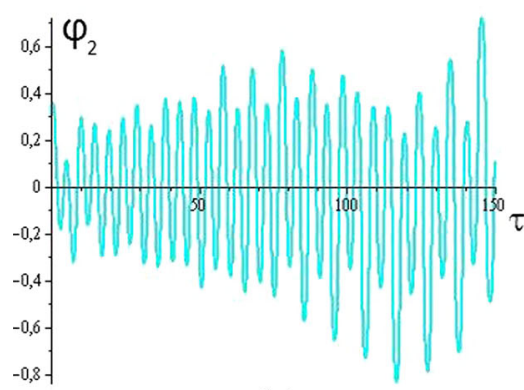

(a)

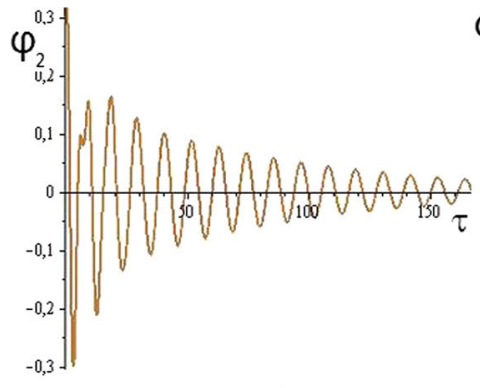

(b)

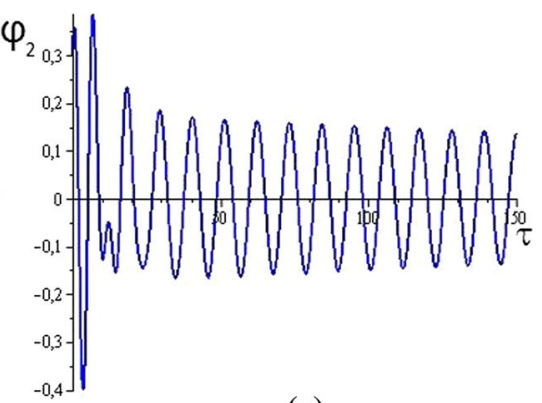

(c)

Fig. 5 Influence of $h$ magnitude on damping rate

Fig. 6 Pendulum with varying length and attached mass

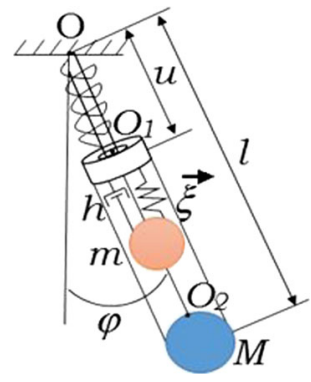

Open Access This article is distributed under the terms of the Creative Commons Attribution 4.0 International License (http:// creativecommons.org/licenses/by/4.0/), which permits unrestricted use, distribution, and reproduction in any medium, provided you give appropriate credit to the original author(s) and the source, provide a link to the Creative Commons license, and indicate if changes were made.

\section{Appendix}

(A) Matrices for example 3:

$$
\begin{aligned}
\boldsymbol{M} & =\left(\begin{array}{ccc}
I_{1}+l^{2}\left(1+m_{a}\right) & l\left(1+m_{a} l_{a}\right) & m_{a} l \\
l\left(1+m_{a} l_{a}\right) & I_{2}+m_{a} l_{a}^{2} & m_{a} l_{a} \\
m_{a} l & m_{a} l_{a} & m_{a}
\end{array}\right), \\
\boldsymbol{K} & =\left(\begin{array}{ccc}
l\left(1+m_{a}\right)+m_{1} l_{1} & 0 & 0 \\
0 & 1+m_{a} l_{a} & m_{a} \\
0 & m_{a} & k
\end{array}\right) .
\end{aligned}
$$

(B) Formulas for example 4: Denoting by $\boldsymbol{r}_{j}$ the radius vector of point $N_{j}(j=1,2)$ in the coordinate system connected with the rigid body, we have

$\boldsymbol{r}_{1}=\left(l+R \sin \alpha, R \cos \alpha_{1}, 0\right)^{T}$,

$\boldsymbol{r}_{2}=(l-R \sin \alpha,-R \cos \alpha, 0)^{T}$.

Kinetic energy of the balancer holds

$$
\begin{aligned}
\mathcal{K}^{+}= & m R^{2}\left[\omega_{1}^{2} \cos ^{2} \alpha+\omega_{2}^{2}\left(\sin ^{2} \alpha+\frac{l^{2}}{R^{2}}\right)\right. \\
& \left.+\omega_{3}^{2}\left(1+\frac{l^{2}}{R^{2}}\right)-\left(\omega_{1} \omega_{2} \sin 2 \alpha+2 \omega_{3} \dot{\alpha}\right)+\dot{\alpha}^{2}\right] .
\end{aligned}
$$

Components of angular velocity vector are given by kinematic Euler relations

$\omega_{1}=\dot{\theta} \cos \varphi+\dot{\psi} \sin \theta \sin \varphi$,

$\omega_{2}=-\dot{\theta} \sin \varphi+\dot{\psi} \sin \theta \cos \varphi$,

$\omega_{3}=\dot{\varphi}+\dot{\psi} \cos \theta$.

The generalized inertia tensor of the system can be written as $\tilde{\boldsymbol{I}}=\boldsymbol{I}+\boldsymbol{I}^{+}$, where $\boldsymbol{I}=\operatorname{diag}\left(I_{1}, I_{2}, I_{2}\right)$ is the inertia tensor of the carrier, and $\boldsymbol{I}^{+}$is the "appendant" inertia tensor of the balancer

$\boldsymbol{I}^{+}=\left(\begin{array}{ccc}2 m R^{2} \cos ^{2} \alpha & -m R^{2} \sin 2 \alpha & 0 \\ -m R^{2} \sin 2 \alpha & 2 m\left(R^{2} \sin ^{2} \alpha+l^{2}\right) & 0 \\ 0 & 0 & 2 m\left(R^{2}+l^{2}\right)\end{array}\right)$.

Potential forces are the gravitational force and elasticity in the balancer's hinge. Hence, the potential energy of the system is given by expression

$\Pi=g\left(M l+2 m l_{b}\right) \sin \theta \sin \varphi+\frac{1}{2} \varkappa\left(\alpha-\alpha_{0}\right)^{2}$.

Here $M$ is the mass of the rigid body, $\alpha_{0}$ is the constant value that corresponds to the equilibrium state of the balancer. For simplicity, we shall take it equal to zero.

The kinetic energy of the system holds

$$
\begin{aligned}
\mathcal{K}= & \mathcal{K}_{0}+\mathcal{K}^{+}=\frac{1}{2}\langle\boldsymbol{\omega}, \tilde{\boldsymbol{I}} \boldsymbol{\omega}\rangle \\
& +m\left\langle\boldsymbol{\omega}, \boldsymbol{r}_{1} \times \dot{\boldsymbol{r}}_{1}+\boldsymbol{r}_{2} \times \dot{\boldsymbol{r}}_{2}\right\rangle+m \dot{\boldsymbol{r}}_{1}^{2},
\end{aligned}
$$




$$
\begin{aligned}
\boldsymbol{B}_{l i n}= & \left(\left[I_{2}-I_{1}+2 m\left(l^{2}-R^{2}\right)\right] \xi_{2}+2 m R^{2} \xi_{3},\right. \\
& \left.-\left[I_{2}+2 m\left(l^{2}+R^{2}\right)\right] \xi_{1}, 2 m R^{2} \xi_{1}\right),\left(\frac{1}{\widetilde{c}_{11}}\right)^{(2)} \\
= & \frac{\left[\left(I_{1}-I_{2}-2 m l^{2}\right) \xi_{1}^{2}+\left(I_{1}-I_{2}+2 m R^{2}-2 m l^{2}\right) \xi_{2}^{2}+2 m R^{2} \xi_{3}^{2}-4 m R^{2} \xi_{2} \xi_{3}\right]}{\left(I_{1}+2 m R^{2}\right)^{2}} \\
\widetilde{\boldsymbol{G}}= & \left(2 I_{2}-I_{1}+4 l^{2}\right)(1,-1,0) .
\end{aligned}
$$

and generalized coordinate $\psi$ is cyclic. The motion Eq. (20) allows the solution

$\theta=\frac{\pi}{2}, \varphi=\frac{\pi}{2}, \alpha=0, \dot{\theta}=0$,

$\dot{\varphi}=0, \dot{\psi}=\omega, \dot{\alpha}=0$,

which describes the permanent rotations of the body with "frozen" balancer around the vertical axis with rotational speed $\omega$.

The square matrix $\widetilde{\boldsymbol{A}}$ is of the third order, $\widetilde{\boldsymbol{B}}$ is a rectangular $1 \times 3$ matrix, and $\widetilde{\boldsymbol{C}}=\widetilde{c}_{11}$ is the scalar one. The elements of these matrices are:

$$
\begin{aligned}
\widetilde{a}_{11}= & \left(I_{1}+2 m R^{2} \cos ^{2} \alpha\right) \cos ^{2} \varphi \\
& +\left[I_{2}+2 m\left(l^{2}+R^{2} \sin ^{2} \alpha\right)\right] \sin ^{2} \varphi \\
& -m R^{2} \sin 2 \alpha \sin 2 \varphi, \\
\widetilde{a}_{12}= & \widetilde{a}_{13}=0, \\
\widetilde{a}_{22}= & I_{2}+2 m\left(l^{2}+R^{2}\right), \widetilde{a}_{23}=-2 m R^{2}, \widetilde{a}_{33}=2 m R^{2}, \\
\widetilde{b}_{11}= & \sin \theta\left\{\frac{1}{2}\left[I_{1}-I_{2}+2 m\left(R^{2} \cos 2 \alpha-l^{2}\right)\right] \sin 2 \varphi\right. \\
& \left.-m R^{2} \sin 2 \alpha \cos 2 \varphi\right\}, \\
\widetilde{b}_{12}= & {\left[I_{2}+2 m\left(l^{2}+R^{2}\right)\right] \cos \theta, \widetilde{b}_{13}=-2 m R^{2} \cos \theta, } \\
\widetilde{c}_{11}= & \left(I_{1}+2 m R^{2} \cos ^{2} \alpha\right) \sin { }^{2} \theta \sin ^{2} \varphi \\
& +\left[I_{2}+2 m\left(l^{2}+R^{2} \sin ^{2} \alpha\right)\right] \sin ^{2} \theta \cos ^{2} \varphi \\
& +\left[I_{2}+2 m\left(l^{2}+R^{2}\right)\right] \cos ^{2} \theta \\
& -m R^{2} \sin 2 \alpha \sin ^{2} \theta \sin ^{2} \varphi .
\end{aligned}
$$

To get linearized equations of the motion, we need further the linear approximation of matrix $\boldsymbol{B}$ and quadratic terms of $\widetilde{c}_{11}$. According to formulas (22), (25) we have
Also the following dimensionless parameters are used

$$
\begin{aligned}
a & =\frac{I_{1}}{I_{2}+2 m l_{1}^{2}}, \quad b=\frac{g\left(M l+2 m l_{1}\right)}{\omega^{2}\left(I_{2}+2 m l_{1}^{2}\right)}, \\
\mu & =\frac{2 m R^{2}}{J_{2}+2 m l_{1}^{2}}, \\
h & =\frac{\hbar}{2 m R^{2} \omega}, \kappa=\frac{\varkappa}{2 m R^{2} \omega^{2}}+1, \tau=\omega t,
\end{aligned}
$$

\section{References}

1. Thomson, W., Tait, P.G.: Treatise on Natural Philosophy, vol. I, part I, New Edition. Cambridge University Press, Cambridge (1879)

2. Chetaev, N.G.: Stability of Motion. Nauka, Moscow (1965). (in Russian)

3. Merkin, D.R.: Introduction to the Theory of Stability. Springer, Berlin (1997)

4. Zajac, E.E.: Kelvin-Tait-Chetaev theorem and extensions. J. Astronaut. Sci. 11, 46-49 (1964)

5. Zajac, E.E.: Comments on "stability of damped mechanical systems and a further extension". AIAA J. 3(9), 1749-1750 (1965)

6. Robertson, R.E.: Notes on the Thomson-Tait-Chetaev stability theorem. J. Astronaut. Sci. 15, 319-324 (1968)

7. Huseyin, K.: Vibrations and Stability of Multiple Parameter Systems. Kluwer Academic Publishers, Dordrecht (1978)

8. Zevin, A.A.: A theory of linear non-conservative systems. J. Appl. Math. Mech. 52, 300-304 (1988)

9. Koshlyakov, V.N., Makarov, V.L.: Mechanical systems, equivalent in Lyapunov's sense to systems not containing non-conservative positional forces. J. Appl. Math. Mech. 71, 10-19 (2007)

10. Chern, S.-J.: Stability theory for linear dissipative Hamiltonian systems. Linear Algebra Appl. 357, 143-162 (2002)

11. Bolotin, V.V., Zhinzher, N.I.: Effects of damping on stability of elastic systems subjected to nonconservative forces. Int. J. Sol. Struct. 5, 965-989 (1969) 
12. Crandall, S.H.: The role of damping in vibration theory. J. Sound Vibr. 11, 3-18 (1970)

13. Crandall, S.H.: The effect of damping on the stability of gyroscopic pendulums. ZAMP Z. Angew. Math. Phys. 46, 761-780 (1995)

14. Hryniv, R., Lancaster, P.: Stabilization of gyroscopic systems. ZAMM Z. Angew. Math. Mech. 81, 675-681 (2001)

15. Agafonov, S.A.: Stability and motion stabilization of nonconservative mechanical systems. J. Math. Sci. 112, 44194497 (2002)

16. Kirillov, O.: Nonconservative stability problems of modern physics. De Gruyter, Berlin (2013)

17. Husein, K., Hagedorn, P., Teschner, W.: On the stability of linear conservative gyroscopic systems. ZAMP Z. Angew. Math. Phys. 34, 807-815 (1983)

18. Kirillov, O.N., Verhulst, F.: Paradoxes of dissipationinduced destabilization or who opened Whitney's umbrella? ZAMM Z. angew. Math. Mech. 90, 462-488 (2010)

19. Pozharitski, G.K.: Characteristic exponents of damped oscillations of mechanical systems with incomplete dissipation. J. Appl. Math. Mech. 29(5), 927-931 (1965)

20. Moran, T.J.: A simple alternative to the Routh-Hurwitz criterion for symmetric systems. ASME J. Appl. Mech. 37, 1168-1170 (1970)

21. Muller, P.C.: Stabilitat und Matrizen. Springer, Berlin (1977)

22. Zhinzher, N.I.: Effect of dissipative forces with incomplete dissipation on the stability of elastic systems. Izv. Ross. Akad. Nauk. MTT 1, 149-155 (1994)

23. Puzyrev, V.E.: The influence of viscous friction on stability of stationary motion of mechanical systems with partial energy dissipation. Dopovidi Nat. Ukr. Ac. Sci. 8, 61-65 (2004). in Russian

24. Bernstein, D.S., Bhat, S.P.: Lyapunov stability, semistability, and asymptotic stability of matrix second-order systems. J. Mech. Design 117, 145-153 (1995)

25. Agafonov, S.A.: On the stability of nonconservative systems with estimation of the attraction domain. J. Dyn. Control Sys. 6(4), 503-510 (2000)
26. Jiang, L.: A generalization of the Barbashin-Krasovski theorem. J. Math. Anal. Appl. 326, 1379-1382 (2007)

27. Tonkov, E.L.: Barbashin-Krasovskii's asymptotic stability theorem in application to control systems on smooth manifolds. Proc. Steklov Inst. Mech. 291, 208-221 (2015)

28. Kalyakin, L.A.: Stability of the autoresonance in a dissipative system. Russ. J. Math. Phys. 23(1), 77-87 (2016)

29. Krasovski, N.N.: Stability of Motion. Stanford University Press, Stanford (1963)

30. Puzyrov, V.E., Topchyi, N.V.: Estimation of the eigenvalues of the linear mechanical system with two degrees of freedom. Mech. Rigid Body 41, 132-140 (2011). in Russian

31. Hagedorn, P., Heffel, E., Lancaster, P., Muller, P.C., Kapuria, S.: Some recent results on MDGKN-systems. ZAMM 95(7), 695-702 (2015)

32. Jekel, D., Hagedorn, P.: Stability of weakly damped MDGKN-systems: the role of velocity proportional terms. ZAMM 97(9), 1128-1135 (2017)

33. Lienard, A., Chipart, M.H.: Sur le signe de la partie reelle des racines d'une equation algebrique. J. Math. Pures Appl. 10(6), 291-346 (1914)

34. Lyapunov, A.M.: The general problem of the stability of motion. Int. J. Control 55, 531-773 (1992)

35. Taylor, J.R.: Classical Mechanics. University Science Books, Sausalito, California (2005)

36. Zhou, Z., Whiteman, C.: Motions of a double pendulum. Nonlinear Anal. Theory Methods Appl. 26(7), 1177-1191 (1996)

37. Nikitina, N.V.: Estimating the chaos boundaries of a double pendulum. Int. Appl. Mech. 47(5), 600-607 (2011)

38. Puzyrov, V.E., Savchenko, N.N.: Using dynamic Vibration absorber for stabilization of a double pendulum oscillations. Nonlinear Dyn. Syst. Theor. 14(4), 402-409 (2014)

39. Pfeiffer, K., Savchenko, A.Ya.: On passive stabilization in critical cases. J. Math. Anal. Appl. 244, 106-119 (2000)

40. Puzyrov, V.E., Kamynina, O.V., Savchenko, N.V.: Use of the passive damper for stabilization of small oscillations of the pendulum with varying length. Bull. Donetsk Nat. Univ. 1-2, 126-131 (2015) 